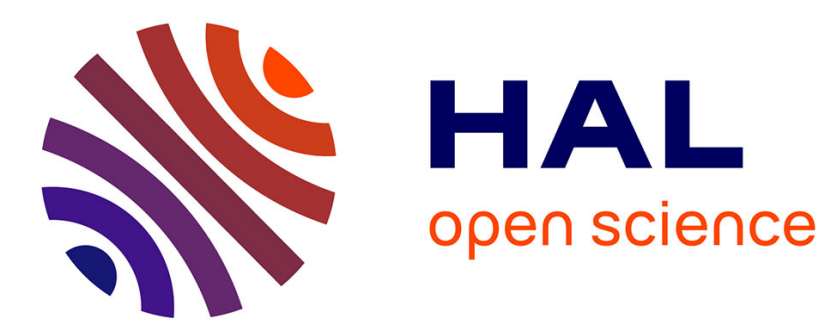

\title{
Presse locale, un média de diversion
}

Loïc Ballarini

\section{To cite this version:}

Loïc Ballarini. Presse locale, un média de diversion. Réseaux: communication, technologie, société, 2008, Médiations. Hommage à Paul Beaud, 26 (148-149), pp.405-426. 10.3166/Réseaux.148-149.405426. hal-03021896

\section{HAL Id: hal-03021896 https://hal.science/hal-03021896}

Submitted on 24 Nov 2020

HAL is a multi-disciplinary open access archive for the deposit and dissemination of scientific research documents, whether they are published or not. The documents may come from teaching and research institutions in France or abroad, or from public or private research centers.
L'archive ouverte pluridisciplinaire HAL, est destinée au dépôt et à la diffusion de documents scientifiques de niveau recherche, publiés ou non, émanant des établissements d'enseignement et de recherche français ou étrangers, des laboratoires publics ou privés. 


\section{PRESSE LOCALE, UN MÉDIA DE DIVERSION}

\section{Loïc Ballarini}

Lavoisier | «éseaux»

2008/2 n $148-149$ | pages 405 à 426

ISSN 0751-7971

ISBN 9782746221901

Article disponible en ligne à l'adresse :

https://www.cairn.info/revue-reseaux1-2008-2-page-405.htm

Distribution électronique Cairn.info pour Lavoisier.

(C) Lavoisier. Tous droits réservés pour tous pays.

La reproduction ou représentation de cet article, notamment par photocopie, n'est autorisée que dans les limites des conditions générales d'utilisation du site ou, le cas échéant, des conditions générales de la licence souscrite par votre établissement. Toute autre reproduction ou représentation, en tout ou partie, sous quelque forme et de quelque manière que ce soit, est interdite sauf accord préalable et écrit de l'éditeur, en dehors des cas prévus par la législation en vigueur en France. Il est précisé que son stockage dans une base de données est également interdit. 


\section{PRESSE LOCALE, UN MÉDIA DE DIVERSION \\ Loïc BALLARINI}




pays de tradition centralisatrice, les journaux régionaux dominent largement les nationaux. Dans un contexte où la problématique de la perte de confiance à l'égard de l'information s'ajoute aux difficultés économiques rencontrées par la presse pour s'adapter à la numérisation et aux nouvelles formes de diffusion des contenus, la presse régionale est souvent vue comme une forme de permanence d'un modèle plus ancien. La proximité, autant physique que symbolique, qu'elle entretient avec ses lecteurs en leur donnant accès au monde le plus directement compréhensible - celui des routines quotidiennes -, la tiendrait à l'écart des difficultés qu'éprouve la presse nationale à rendre compte des phénomènes de mondialisation et de l'éloignement croissant des centres de décision politiques et économiques.

Mais cette explication ne saurait suffire: la presse régionale française, loin d'échapper à la crise, ne cesse de perdre des lecteurs. Parallèlement à d'autres travaux sur la réception ou les pratiques et représentations des professionnels, cet article entend interroger le contenu même de la presse régionale. Le moyen en est une analyse de contenu de la presse régionale de Bretagne. Celle-ci révèle un décalage croissant entre le monde que la presse locale donne à lire et celui que vivent ses lecteurs. Malgré une diversité et une neutralité apparentes, l'information locale entretient les hiérarchies, est volontiers complaisante et demeure marquée par une vision du monde qui privilégie la stabilité sociale. D'un monde fait d'interrelations et de processus dynamiques, elle ne retient qu'une juxtaposition de micro-événements sans contexte. Nos travaux confirment et renforcent ainsi ce que nombre de chercheurs supposent ou postulent. Occupant une position dominante sur un territoire qu'elle dit refléter, médiateur très particulier d'un espace public partiel, la presse régionale détourne le regard de la profondeur et de la diversité de la société pour l'attirer sur une construction des rapports sociaux simplifiée à l'extrême.

Le modèle de presse régionale que nous connaissons aujourd'hui, basé sur une information locale destinée au public le plus large, s'est construit à partir de la seconde moitié du XIX ${ }^{\mathrm{e}}$ siècle, et s'est imposé à la fin des années 1930, 
date à laquelle les ventes des quotidiens de province ont dépassé celles des titres édités à Paris. La presse régionale, qui parvient même à sortir plus forte de la Seconde Guerre mondiale qu'elle n'y est entrée, continue ensuite à creuser l'écart avec la presse nationale, en adoptant notamment des méthodes de gestion plus efficaces et en s'ouvrant largement à la publicité, à laquelle elle habitue les futurs téléspectateurs et lecteurs de magazines.

A la fin des années 1960 cependant, des critiques de plus en plus virulentes sont portées à son encontre, sans que les réponses qu'elle apporte n'apparaissent satisfaisantes. Accusée de conservatisme et de conformisme, elle réagit en diminuant la place accordée aux décorations et commémorations, et en augmentant celle de la vie économique et sociale, des associations, des équipements. Cela n'empêche pas une baisse de son lectorat de s'enclencher à la fin des années 1970. Elle est certes lente, mais continue. Et même si leur diffusion reste trois fois supérieure à celle des quotidiens nationaux, les quotidiens régionaux sont revenus aujourd'hui à leur niveau de $1950^{1}$.

Les mouvements de concentration économique, qui sont certainement le phénomène majeur d'évolution de cette forme de presse depuis trente ans, ne sont pas parvenus à enrayer cette baisse - mais ils ont contribué à diviser par trois le nombre de titres : il y avait 175 quotidiens régionaux et départementaux à la Libération, il en restait 60 en $2005^{2}$. La presse hebdomadaire régionale a quant à elle continué à créer des titres et à gagner des lecteurs jusqu'à la fin des années 1990. Mais cette illusion d'un secteur à part de la presse régionale ne tient plus aujourd'hui : sa diffusion a baissé de $5 \%$ depuis 2000 .

Les difficultés de la presse régionale française apparaissent à une période cruciale de l'évolution des territoires, caractérisés au cours de la seconde moitié du $\mathrm{XX}^{\mathrm{e}}$ siècle par une profonde complexification. A la fin des années 1950 sont créés les plans d'aménagement du territoire et les «régions de programme », qui préfigurent les régions administratives définies en 1982. En 1992 sont créées les communautés de communes, puis en 1999 les «pays » qui les regroupent, créant ainsi un espace intermédiaire entre commune et

1. Tirage moyen des quotidiens régionaux en $1952: 6,18$ millions par jour (source ALBERT, s.d.a). Ventes moyennes des quotidiens régionaux en $2005: 5,58$ millions par jour (source OJD, 2006a et 2006b). Si l'on tient compte du taux d'invendus relativement faible de la presse régionale, les deux chiffres peuvent être considérés comme équivalents.

2. Pour le chiffre de 1946, cf. ALBERT s.d.b. Pour le chiffre de 2005, cf. DDM, 2007,

p. $117: 69$ quotidiens locaux en 2005 , dont 9 publiés outre-mer. 
département, censé mieux correspondre aux bassins de vie de la population. Aujourd'hui, sans compter les syndicats intercommunaux à vocation unique ou multiple (pour la gestion des eaux, la collecte des ordures ou l'entretien d'une forêt), le mille-feuilles administratif français compte neuf couches allant de la commune à l'Europe, dont sept ont en charge le découpage du niveau «local»: commune, canton, intercommunalité ${ }^{3}$, arrondissement, «pays », département, région. A ces territoires « objectifs » s'ajoutent les appartenances familiales, amicales, professionnelles, associatives, politiques, religieuses, etc.

Dans la société qui a vu naître la presse, on appartenait le plus souvent à une famille et un village, parfois à une corporation. Dans celle qui assiste au développement d'internet, il est quasiment impossible de faire la liste des différents territoires auxquels chacun appartient ou peut se sentir appartenir. Le sentiment d'appartenance a ici toute son importance, tant le territoire et le local sont en eux-mêmes ambigus. Selon le point de vue, on peut aussi bien les considérer comme inertes et lents que comme réactifs et avant-gardistes. Isabelle Pailliart situe ainsi le local « à l'entrecroisement de deux dimensions : celle qui permet d'être plus innovant parce que l'on est situé dans un espace micro, celle d'être également, parce qu'étant la partie d'un tout, le révélateur des tensions qui affectent le tout. Ce double caractère du territoire conduit à mettre l'accent tantôt sur la dimension novatrice, tantôt sur la dimension conservatrice. $»^{4}$ D'autant plus délicat à saisir que ses limites sont constamment repoussées et ses structures sans cesse plus complexes, le territoire possède ce qu'Isabelle Pailliart décrit comme une «originalité fondamentale: il n'existe que parce qu'il est dans une situation de continuelle production de lui-même, les médias participant étroitement de cet incessant mouvement. $»^{5}$

Passer à côté du double caractère et de l'originalité fondamentale du territoire, rester sur l'une ou l'autre dimension conduirait à figer les processus dynamiques de sa constitution et de son évolution. C'est pourtant ce qui semble se produire avec la presse régionale. Au cours de son premier siècle d'existence, elle a en effet entretenu une relation très étroite avec les évolutions sociales et économiques du monde dans lequel elle s'insère et qu'elle prétend expliquer. Les travaux historiques montrent bien que la presse de province a évolué avec la société, chacune s'inspirant de l'autre autant

\footnotetext{
3. Communautés de communes, communautés d'agglomération et communautés urbaines (l'appellation varie selon la taille)

4. PAILLIART, 1994, p. 65-66.

5. PAILLIART, 1994, p. 247.
} 
qu'elle l'inspirait ${ }^{6}$. Mais, depuis le dernier quart du $\mathrm{XX}^{\mathrm{e}}$ siècle, la presse connaît la plus longue et la plus importante crise de son histoire. Comme si la société avait continué d'évoluer de son côté, tandis que la presse peinait à se renouveler. La vision de la proximité à partir de laquelle la presse régionale a défini sa manière de traiter l'information n'est-elle pas devenue une construction trop éloignée de la réalité de la vie de ses lecteurs potentiels?

\section{UNE INFORMATION APPAREMMENT DIVERSIFIÉE}

\section{Quatre villes, quatre journaux}

Pour tenter de répondre à cette question, il nous faut comprendre quel est le monde que la presse locale donne à lire. Il ne s'agit pas ici de s'intéresser à ce que veulent dire les journalistes et les patrons de presse, ni de mener une étude de réception, qui est une étape ultérieure de ce travail, mais d'analyser le contenu de la presse régionale. Par presse régionale, nous entendons les journaux d'information généraliste, quotidiens et hebdomadaires, publiés dans une échelle qui va de la commune à la région. Les journaux institutionnels et spécialisés, qu'ils soient corporatistes ou de petites annonces par exemple, en sont donc exclus. Ce qui donne une vingtaine de titres pour la Bretagne, région choisie pour cette étude parce qu'elle est, depuis le début $\mathrm{du} \mathrm{XX}^{\mathrm{e}}$ siècle, une des régions françaises les plus dynamiques en matière de presse locale, et que son paysage y est encore contrasté. On y trouve en effet aussi bien le plus important quotidien français en termes de diffusion que de petits hebdomadaires indépendants. Au sein de cette région, nous avons retenu quatre villes. Sur une période d'une semaine pour les quotidiens et d'un mois pour les hebdomadaires ${ }^{7}$, nous avons relevé tous les articles concernant ces communes, et analysé leur emplacement dans le journal, leur taille, thème, type (information-service, portrait, enquête, etc.), auteur et ton, ainsi que les photos et citations utilisées. Nous reprenons ici les principaux résultats de cette analyse de contenu, dont le corpus compte 1651 articles.

\footnotetext{
6. Notamment MARTIN, 2002 ; CHARON, 2003 ; JEANNENEY, 1998.

7. Le moment de l'année a été choisi pour correspondre à une période d'activité diversifiée de la presse régionale (politique, associations, économie, etc.), sans domination d'un thème ou d'un événement particulier.
} 
Les quatre villes de l'étude ont été choisies en fonction de leur diversité en matière économique, sociale, démographique et médiatique ${ }^{8}$. Il s'agit de :

Rennes : 206000 habitants en 1999, préfecture d'Ille-et-Vilaine et capitale régionale. Son économie est diversifiée (industrie, services, NTIC bien implantées). Elle est le siège du quotidien Ouest-France, qui y dispose du monopole sur l'information écrite,

Brest (150 000 habitants), sous-préfecture du Finistère, est la seconde ville de la région, dans l'ombre de Rennes. Dominée par la présence de l'arsenal militaire auquel on l'identifie souvent, elle est pourtant très active, notamment au plan culturel, mais peine à trouver une reconnaissance. Fief historique du quotidien Le Télégramme, elle n'échappe cependant pas à la concurrence d'Ouest-France. Un hebdomadaire indépendant, Le Progrès de Cornouaille/Courrier du Léon, y est également diffusé,

Saint-Brieuc (50 000 habitants) est la préfecture des Côtes-d'Armor. C'est une ville moyenne au passé industriel fort, qui peine à se reconvertir. Elle est l'exemple d'une ville-centre historique dont les commerces connaissent des difficultés récurrentes au profit d'une périphérie florissante. Ouest-France et Le Télégramme s'y affrontent, l'hebdomadaire Le Penthièvre s'y développe depuis sa création en 2000.

Quintin (2 900 habitants), enfin, est un chef-lieu de canton situé dans le sud de la zone d'influence de Saint-Brieuc. Ancienne capitale de la fabrication et du négoce des toiles de lin dites «Bretagnes », elle est restée une petite ville relativement autonome, qui bénéficie aujourd'hui des mouvements de « rurbanisation». Elle appartient à la tranche des communes de 1000 à 5000 habitants dans laquelle vivent $41 \%$ des Bretons (moyenne nationale 24 \%). Ouest-France, Le Télégramme et Le Penthièvre y sont présents.

Dans ces quatre villes, on rencontre quatre quotidiens et hebdomadaires régionaux :

Ouest-France. Créé en 1944, c'est le descendant de l'Ouest-Éclair, fondé à Rennes en 1899. Avec une diffusion stabilisée autour de 781000

8. Avec l'aide du diagnostic préalable à l'établissement du Schéma régional d'aménagement et de développement du territoire de la région Bretagne, SRADT: Bretagne 2015, Conseil régional de Bretagne, octobre 2002. 
exemplaires par jour, soit plus que Le Monde et Le Figaro réunis, OuestFrance est le premier quotidien français depuis 1976. C'est le navire amiral du groupe Sipa, qui compte aussi, entre autres, Ouest-France Multimédia, Publihebdos (57 hebdomadaires dont Le Penthièvre), Sofiouest (les 160 gratuits d'annonces de Spir Communications, une maison d'édition, etc.), $50 \%$ de 20 Minutes SA (qui édite le quotidien gratuit 20 Minutes en France), et désormais les quotidiens anciennement édités par la Socpresse dans l'ouest. Particularité : le groupe Sipa est depuis 1990 filiale à 99,91\% d'une association loi 1901, l'Association pour le soutien des principes de la démocratie humaniste. Son bureau est soigneusement verrouillé, empêchant ainsi qui que ce soit de prendre le contrôle du groupe,

Le Télégramme. Comme Ouest-France, Le Télégramme a été fondé en 1944, à la suite de La Dépêche de Brest qui datait de 1886. C'est aussi un journal qui appartient à la même famille depuis ses débuts ou presque. Bloqué dans son développement par la puissance d'Ouest-France, il a tout de même réussi à s'étendre aux Côtes-d'Armor et au Morbihan. Surtout, il a su développer ses ventes à l'intérieur de son territoire historique, par exemple en étant le premier quotidien français à adopter la couleur en 1967. Le Finistère est ainsi un des départements où le taux de pénétration de la presse quotidienne régionale est le plus fort,

Le Télégramme a atteint un pic de diffusion en 1991 avec 215000 exemplaires par jour, mais il est retombé à 194000 en 2002. Le journal a alors changé de maquette. Il est désormais au format tabloïd, agrafé, et met l'information locale plus en valeur grâce notamment à une seconde "une » spécifique à chaque édition, à l'intérieur du journal. Il dépassait les 206000 exemplaires en 2006. Le Télégramme possède également quelques hebdomadaires, une maison d'édition, des parts dans la télévision locale Nantes 7 et une régie publicitaire,

Le Progrès de Cornouaille/Le Courrier du Léon. Il s'agit de deux hebdomadaires au contenu identique, et dont seul le nom diffère. Le Progrès de Cornouaille est publié au sud du Finistère, Le Courrier du Léon au nord. Il en va ainsi depuis 1895, date à laquelle deux feuilles créées par l'évêché de Brest (Le Courrier de Cornouaille et Le Courrier du Finistère) ont fusionné. Ayant été interdites à la Libération, elles ont simplement changé de nom pour reparaître, leur gestion ayant été transférée à des laïcs via la Société des éditions nouvelles du Finistère, qui est toujours indépendante aujourd'hui. Le contenu du Progrès/Courrier est assez original, puisqu'il n'est pas découpé 
géographiquement comme les autres journaux locaux, mais par thèmes (Pêche, Mémoire, Santé...). Ces thèmes ne reviennent pas nécessairement toutes les semaines, mais en fonction de l'actualité. Le journal vend aujourd'hui environ 7000 exemplaires chaque semaine, contre un tirage de 30000 en 1926,

Le Penthièvre. "L'hebdo du Pays de Saint-Brieuc » a remplacé en juin 2000 le bulletin bimensuel L'Avis local, qui venait d'être racheté par le groupe Publihebdos, filiale d'Ouest-France. C'est le sixième hebdomadaire lui appartenant dans les Côtes-d'Armor, ce qui permet au groupe de couvrir la totalité du territoire de ce département de 540000 habitants. En 2006, Le Penthièvre vendait en moyenne 5000 exemplaires par semaine (18\% de plus qu'en 2002).

\section{Types d'articles}

Sans grande surprise, les comptes-rendus forment près de la moitié du corpus (graphique 1), avec 789 items, soit $47,8 \%$ du total. La moitié de ces comptesrendus sont des articles, un gros quart sont des brèves, le reste se répartissant en faits divers, encadrés, photo-légendes, comptes-rendus d'audience et nécrologies. Les informations-service constituent le deuxième type d'article le plus utilisé $(352,21,3 \%)$. Si on leur ajoute les annonces de spectacle ou d'événements divers $(211,12,8 \%)$, on obtient un pôle d'un tiers d'articles d'informations pratiques permettant d'organiser son emploi du temps. Aucun autre type d'articles ne dépasse les $5 \%$. On peut cependant regrouper reportages, entretiens, portraits et analyses, qui ont en commun de demander une démarche d'approfondissement de la part du journaliste. Ensemble, ils représentent alors $10,6 \%$ du total des articles. Il ne faut pourtant pas dissimuler la part des plus réduites consacrée aux articles d'analyse. Seuls 9 $(0,5 \%)$ peuvent être considérés comme des mises en perspective fouillées d'un sujet, avec rappel du contexte et présence de plusieurs points de vue. Leur emplacement dans le journal ne manque pas non plus d'étonner: ces articles « exemplaires» se trouvent tous en sports, où l'annonce de matches importants nécessitait de revenir sur le déroulement de la saison, d'interroger des représentants de chaque club et d'étudier leurs points faibles et leurs atouts. Le « journalisme d'investigation » n'est pas toujours là où l'on croit...

Les rappels (75, 4,5\%, pour moitié accompagnés de photos), même s'ils occupent beaucoup moins de place dans le journal, sont tout de même plus nombreux que les reportages. Leur vocation est d'organiser la circulation à 
l'intérieur du journal, en renvoyant le lecteur d'une page à une autre, d'un simple titre parfois accompagné de quelques lignes vers le développement complet de l'information. Or cette circulation comporte deux étages qui ne communiquent pratiquement jamais. Les liens qui mènent aux pages locales se trouvent au niveau du département ou de la région. La une, quant à elle, a une ambition clairement nationale: elle renvoie quasi-exclusivement aux pages nationales et internationales, ne laissant que très peu de place à l'information locale. Autrement dit, on lit les informations locales par habitude ou par principe, mais pas parce qu'une démarche journalistique ou éditoriale nous y invite. La situation est différente dans les hebdomadaires, surtout Le Penthièvre, où de nombreuses communes sont présentes en une - mais ces journaux, contrairement aux quotidiens, ne traitent pas du tout l'actualité nationale ou internationale. On remarque enfin que le journal local donne peu la parole à ses lecteurs $(38,2,4 \%)$.

\section{Graphique 1. Répartition des articles par type}

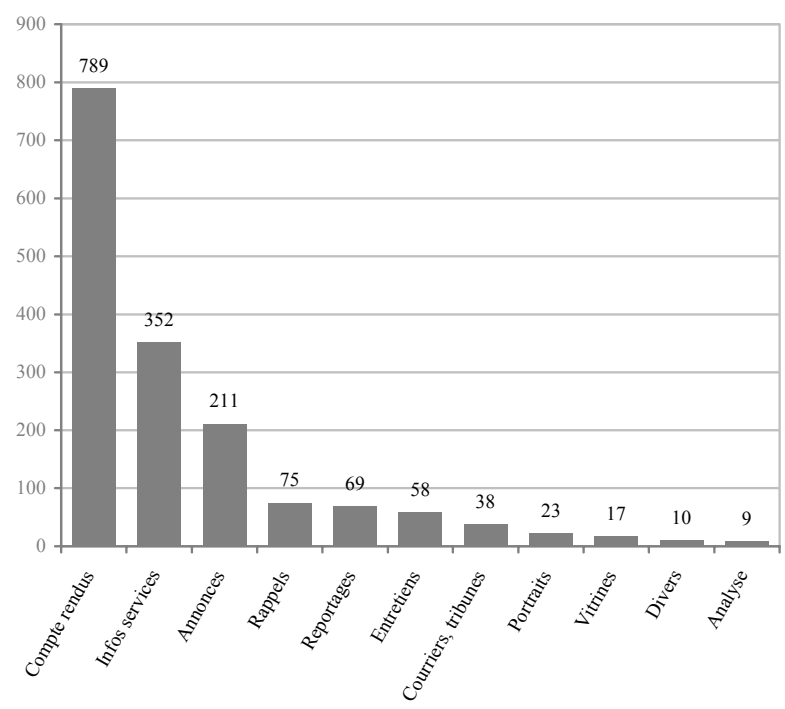




\section{Auteurs des articles}

\section{Graphique 2. Répartition des articles par auteur}

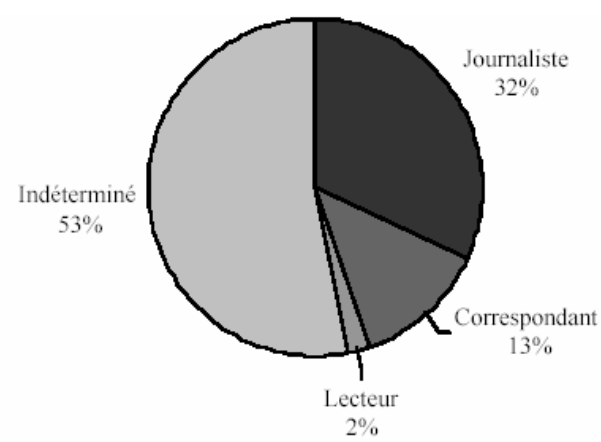

La question de savoir qui écrit dans le journal (graphique 2) pourrait paraître saugrenue. Elle mérite pourtant d'être posée, ne serait-ce que pour constater que dans plus de la moitié des cas, il est impossible de savoir qui est l'auteur d'un article ${ }^{9}$. Nous conviendrons que cette indétermination demeure sans conséquence pour les informations-service. Ne pas connaître l'auteur des horaires de permanences et d'ouverture des administrations, des dates des prochains bals et de la liste des gagnants de la tombola importe peu. Mais ces éléments ne représentent qu'un cinquième de notre corpus. Sans conséquence encore, le fait de ne pouvoir trancher entre journaliste et correspondant $^{10}$ à la lecture des nombreuses photo-légendes des rubriques « ville », qui concernent en général des événements de peu d’importance décrits très factuellement. La question de la distance, voire du recul critique que tous les journaux revendiquent, se pose par contre de façon plus délicate

\footnotetext{
9. On le sait quand les articles sont signés, ou qu'ils ne peuvent être à l'évidence que le fait d'un journaliste (interview d'une personnalité par exemple) ou d'un correspondant (compterendu d'une soirée festive dans une petite commune ou un quartier).

10. Les correspondants dont il est ici question ne le sont pas au sens des correspondants à l'étranger des grands journaux ou agences de presse. Il s'agit de correspondants locaux de presse (CLP), tels que définis par l'article 10 de la loi du 28 janvier 1987. Les CLP ne sont pas des journalistes professionnels, mais des personnes exerçant souvent un autre métier et écrivant une part en général déterminante du contenu local des journaux régionaux, sans contrat de travail et pour une rémunération dérisoire.
} 
dans le cas des annonces de spectacle ou d'expositions. Elles sont en effet pour la plupart constituées d'extraits de dossiers de presse plus ou moins bien recopiés, affirmant l'innovation et le caractère inédit des propositions, ainsi que le talent évidemment confirmé des artistes. En ce qui concerne les inaugurations de bâtiments ou les mises en place de nouveaux services, il peut être très gênant de ne pas savoir si leur contenu provient bien d'un travail journalistique, ou plus directement des chargés de communication qui promeuvent ces « événements ».

Un exemple tiré de notre corpus illustre bien les dérives de sens auxquelles cette indétermination peut mener. Dans son édition du 25 janvier 2005, Le Télégramme consacre un quart de page à deux navires militaires, dont le port d'attache est Brest, qui sont alors en opération à Sumatra afin de venir en aide aux victimes du tsunami de décembre 2004. Dans l'article, qui est tout à la gloire de la marine française, des propos d'officiers et de marins sont retranscrits. Mais aucune mention n'est faite de leur origine : s'agit-il d'un communiqué officiel de la marine, d'une interview téléphonique, du reportage d'un envoyé spécial, voire du témoignage de soldats déjà rentrés au pays? L'article se termine par ces phrases, qui ne sont pas présentées comme une citation mais qui en ont toute la saveur: "Inutile de donner la réplique à ceux qui, dans l'atmosphère ouatée de leur bureau, ont reproché aux marins français d'intervenir aussi tardivement. Le soutien humanitaire ne fait que commencer» Quelle valeur peut-on accorder à ces propos dont l'auteur, journaliste ou officier, n'est pas identifiable ? Comment lire, comprendre et interpréter un article dont on ne connaît pas l'origine et qu'on ne peut replacer dans son contexte informationnel ?

\section{DIVERTIR, INFORMER, INTÉGRER...}

De quoi parlent ces 1651 articles ? La vie locale telle qu'elle est décrite par la presse régionale bretonne se divise en 11 thèmes (graphique 3). Le premier d'entre eux est celui des services, apportés par de courtes informations pratiques, souvent rédigées en style télégraphique. Vient ensuite le sport, qui domine largement la politique, laquelle regroupe pourtant la vie de toutes les institutions de la commune à l'Europe, l'action militante des partis et associations, et les deux principaux champs d'action que sont l'aménagement urbain et la vie des quartiers, dans laquelle nous incluons les maisons et conseils de quartier, les procédures de type médiation de voisinage et l'action des associations de riverains. La culture 
est la seule autre catégorie à passer la barre des $10 \%$ d'articles. La fête, sous toutes ses formes, occupe encore une place importante. L'action sociale et les solidarités dépassent à peine les $7 \%$, talonnés par la sécurité. Le monde du travail (de l'installation de nouveaux commerces aux conflits sociaux) dépasse encore les $5 \%$. Divers thèmes peu représentés, l'état civil et l'autopromotion du journal ferment la marche, cette dernière catégorie, qui regroupe les créations de nouvelles rubriques ou les animations organisées par le journal, constituant tout de même 1,6\% des articles.

Graphique 3. Les onze thèmes de la vie locale

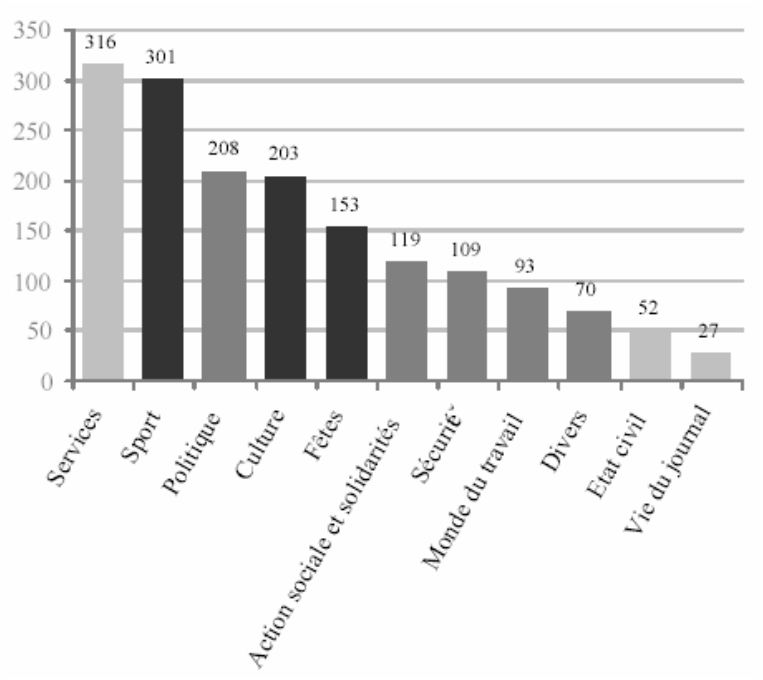

Ces résultats, qui corroborent ceux d'autres études du même type ${ }^{11}$, montrent une vie locale aux préoccupations renversées par rapport à la vie quotidienne d'un Français plus ou moins «moyen ». Le travail, qui occupe environ un quart de notre vie - sans compter les conversations qui portent sur lui hors de son cadre -, ne représente qu'à peine plus d'un vingtième du

11. Notamment RINGLET, 1981. 
journal. Alors que le sport, à qui les plus courageux consacrent au mieux une dizaine d'heures par semaine (1/15 de leur temps), occupe un cinquième du journal. Il est également étonnant que les thèmes de l'environnement, de la santé et de l'éducation, qui possèdent pourtant d'importants enjeux locaux, soient si peu abordés par les journaux qu'il ait fallu les regrouper en « divers » pour les rendre visibles.

\section{Graphique 4. Les trois fonctions du journal local}

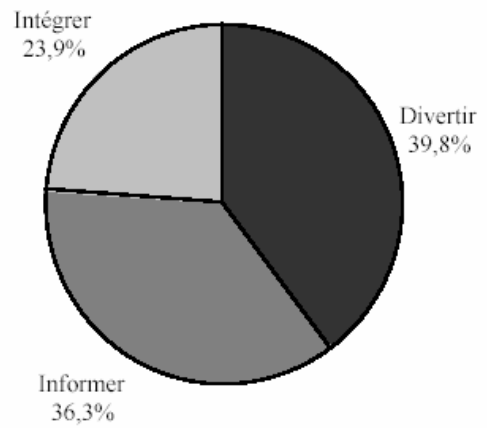

De ces 11 thèmes, on peut dégager trois grandes fonctions du journal local (graphique 4). La fonction dominante apparaît être celle du divertissement $(39,8 \%)$. Elle regroupe le sport, qui est, jusque dans la compétition amateur, une pratique de loisirs, ainsi que la culture et la fête. La seconde fonction du journal local est l'information (36,3\%). Politique, action sociale, sécurité, monde du travail et divers autres thèmes concourent ainsi à éclairer le monde auquel appartiennent les lecteurs. Enfin, le journal local a pour troisième mission l'intégration des lecteurs, en mettant à leur disposition des services, en les renseignant sur l'état civil... et en les incitant à rester fidèle à leur journal $(23,9 \%)$.

\section{... AVEC LE SOURIRE !}

Pour mesurer le ton des articles, la façon dont les choses sont décrites, nous nous sommes inspiré d'une analyse de sites internet d'information locale 
(guides de villes et sites municipaux) réalisée par Denis Ruellan ${ }^{12}$. «Sur un axe qui va de l'engagement politique à l'objet marchand en passant par la neutralité du service $»^{13}$, Denis Ruellan distinguait dix manières de traiter l'information. Nous avons adapté et transformé cette échelle pour qu'elle puisse s'appliquer, non plus à un site ou un journal en général, mais à des articles particuliers, que nous classons pour notre part en huit catégories décrivant leur « ton ».

- Engagé : contient une prise de position politique de l'auteur de l'article.

- Critique : s'articule autour d'une critique, en général dirigée contre une institution.

- Distancié : caractérisé par la présence d'au moins deux points de vue dans l'article, ou par une démarche de l'auteur visant à remettre le sujet dans un contexte plus général. Dans le cas particulier du compte-rendu de séance d'une assemblée politique type conseil municipal, où plusieurs opinions s'expriment de façon quasi-systématique, la distanciation sera marquée par l'apport par le journaliste d'un recul supplémentaire. Sinon l'article appartient à la catégorie suivante.

- Clinique: description des faits, souvent minutieuse, qui n'est accompagnée d'aucun jugement ni remise en contexte.

- Pratique : domaine de l'information-service, brève et sans fioritures, regroupant les horaires des permanences politiques et de services administratifs, les annonces de réunions du club du troisième âge ou de la ludothèque, les animations à venir, etc.

- «Perroquet»: article dans lequel l'auteur reprend manifestement à son compte les dires d'une autre personne sans user des guillemets, sans apporter de contradiction ni de mise en contexte. Cas fréquent des annonces de spectacles dans lesquelles le journaliste se contente de recopier le dossier de presse.

- Valorisant : «Dire du bien de ceux qui font du bien au local » ${ }^{14}$, ainsi peut se définir ce style mettant en valeur des personnes, des institutions, voire des entreprises. Est également considérée comme valorisante toute interview qui ne comprend pas de questions "gênantes" et aide l'interlocuteur à se présenter sous son meilleur jour.

12. RUELLAN, 2001, p. 214-217.

13. RUELLAN, 2001, p. 214.

14. RUELLAN, 2001, p. 215. 
- Promotionnel : autre forme de mise en valeur, cette fois dans le cadre d'un partenariat, voire sur simple demande des personnes présentées dans l'article, comme dans le cas des « vitrines » au statut ambigu. L'ouverture d'un magasin constitue certes une «information», mais lui consacrer un article élogieux revient en effet à une publicité déguisée. Cette catégorie contient également l'autopromotion par laquelle le journal se met lui-même en valeur.

- Divers : rares articles pédagogiques, mises en garde contre de faux démarcheurs, pavés d'humour de fin de semaine.

\section{Graphique 5. Répartition des articles par ton}

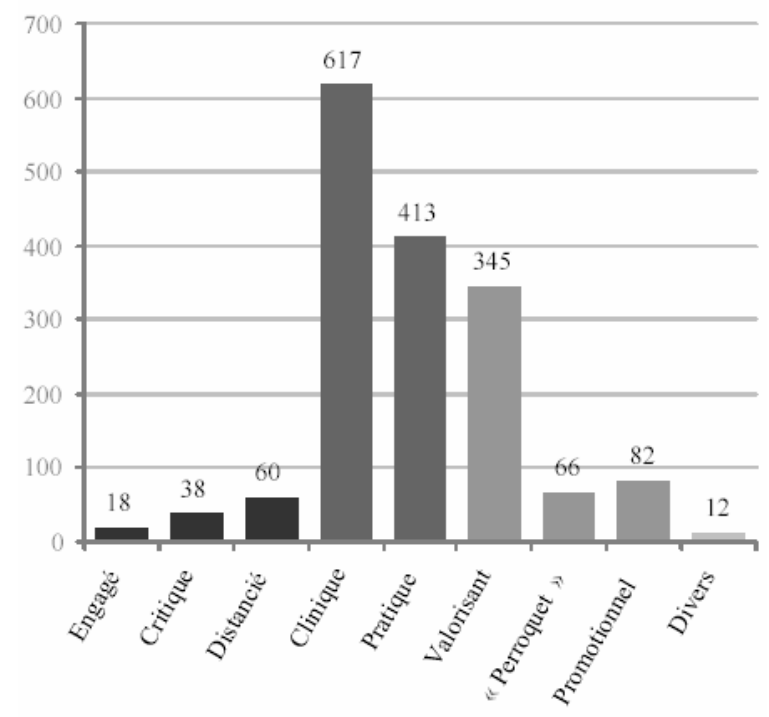

La répartition des articles selon leur ton (graphique 5) révèle une très nette prédominance des catégories clinique et pratique (617 et 413 occurrences). $62,4 \%$ du contenu du journal sont donc «neutres » ou réputés tels. C'est une large majorité, mais c'est aussi en-dessous de ce à quoi l'on aurait pu s'attendre. En effet, une bonne partie de ces articles neutres sont des 
informations-services qui ne peuvent guère adopter un autre ton. De plus, les articles engagés ou critiques sont quasi-inexistants $(3,4 \%$ du total), ce qui confirme la tendance engagée à la fin du XIX ${ }^{\mathrm{e}}$ siècle de vouloir parler à tous et de réfréner les engagements politiques des journaux. Si les journaux locaux ne sont donc pas aussi neutres qu'on aurait pu l'attendre, c'est en fait parce qu'ils semblent s'être donnés pour mission de valoriser « ceux qui font bouger le pays». Les articles "perroquet», valorisants ou promotionnels représentent en effet $29,9 \%$ du total ! Il s'agit là d'une tendance largement surreprésentée, alors qu'en face, la pratique de la distanciation, dont chaque journaliste s'enorgueillit, n'atteint pas les $4 \%$. On peut donc aborder beaucoup de sujets dans un journal local, surtout s'il s'agit de divertissement et d'information-service, et de préférence avec le sourire.

\section{LA PERMANENCE DES HIÉRARCHIES}

Les citations et photographies de personnes ou de documents peuvent être réparties en 13 catégories, comme on le voit dans le tableau 1.

En ce qui concerne les citations, les vedettes incontestées du journal local sont les sportifs, devant les membres d'associations et les politiques. On trouve par contre beaucoup d'artistes sur les photos, mais assez peu de politiques. On interroge un nombre important de professionnels divers - deux fois plus que les simples particuliers présentés sans qualité. Ces interlocuteurs, appelés à témoigner en fonction de leurs compétences professionnelles, sont peut-être le pendant local des experts que chérissent les médias nationaux, et qui ne représentent qu'à peine $1 \%$ de notre corpus. Globalement, ces deux classements appellent la même remarque que les thèmes abordés par la presse locale : la répartition des sources citées et photographiées est très éloignée de la réalité de la vie sociale. Dans le cas des citations, cela se double par une très forte inégalité à l'intérieur des catégories, où les dirigeants, quelle que soit leur nature, dominent largement. Ainsi, on donne bien plus souvent la parole au président de l'association qu'au bénévole, au chef d'entreprise qu'à l'employé, au maire qu'au conseiller municipal. Il y a plus de deux entraîneurs pour un joueur dans la presse locale, plus de deux présidents pour un bénévole, et ce rapport passe à plus de cinq si l'on considère tous les responsables associatifs (membres du bureau ou du conseil d'administration, organisateurs, directeurs, membres du jury). Le maire et ses adjoints sont 3,5 fois plus nombreux que les conseillers municipaux et les chefs d'entreprise dix-huit fois plus que leurs employés. Le déséquilibre interne aux catégories disparaît par contre sur les 
photos : on repasse à deux bénévoles pour un président, et à douze joueurs pour un entraîneur, ce qui est plus pratique pour disputer un match de football, sport favori du journal local. Mais au vu de ce qui précède, on peut douter que cela soit dû à un souci d'équité. Il y a en effet de manière générale beaucoup plus de monde sur les photos que de personnes citées dans les articles, et cela pour une raison simple: il faut vendre des journaux à tous ceux que l'on peut reconnaitre, à leur famille et à leurs amis.

Tableau 1. Sources citées dans les articles et représentées sur les photos

\begin{tabular}{|l|rr|rr|}
\cline { 2 - 5 } \multicolumn{1}{c|}{} & \multicolumn{2}{c|}{ citations } & \multicolumn{2}{c|}{ photos } \\
\cline { 2 - 5 } \multicolumn{1}{c|}{ nb } & $\%$ & nb & $\%$ \\
\hline Sportifs & 205 & 20,6 & 196 & 18,1 \\
\hline Associatifs & 194 & 19,5 & 167 & 15,4 \\
\hline Hommes et femmes politiques & 151 & 15,2 & 86 & 8,0 \\
\hline Professionnels & 109 & 11,0 & 73 & 6,8 \\
\hline Artistes (professionnels ou non) & 79 & 8,0 & 180 & 16,7 \\
\hline Administrations et institutions & 74 & 7,5 & 34 & 3,1 \\
\hline Particuliers & 52 & 5,2 & 132 & 12,2 \\
\hline Entreprises & 45 & 4,5 & 45 & 4,2 \\
\hline Documents & 31 & 3,1 & 9 & 0,8 \\
\hline Divers & 24 & 2,4 & 82 & 7,6 \\
\hline Étudiants & 17 & 1,7 & 57 & 5,3 \\
\hline Experts et personnalités & 10 & 1,0 & 17 & 1,6 \\
\hline Le journal lui-même & 2 & 0,2 & 3 & 0,3 \\
\hline & 993 & 100 & 1081 & 100 \\
\hline
\end{tabular}

Alors certes, la presse d'aujourd'hui n'est plus la presse des notables qui dominait il y a un siècle : elle aborde beaucoup de sujets, fait appel à beaucoup de sources différentes, sans que ses pages ne soient encombrées par des références aux élus locaux. Mais, derrière cette apparente diversité, elle organise une autre forme de notabilisation. Le cercle des édiles s'est élargi aux chefs d'entreprise, aux responsables associatifs, aux entraîneurs sportifs, qui forment une sorte d'élite locale dont on ne sort pas ou très rarement. On peut ici reprendre ce que dit Jacques Le Bohec des relations entre journalistes localiers et élus politiques: "Dans de nombreuses configurations de jeu, il existe une sorte de modus vivendi qui conduit édiles et localiers à s'aménager un espace symbolique clos où ils vont s'entre-célébrer comme membres d'une 
« élite» spatialement circonscrite. [...] Ce jeu restreint, qui implique un faible nombre de personnes, interdit de s'exprimer en termes d'"espace public local" pour décrire la vie $[\ldots]$ dans la plupart des communes. » 15

Cette forme de fermeture de la presse locale est encore accentuée par sa façon de traiter, ou plutôt de ne pas traiter l'échec. Sur 993 personnes citées dans notre corpus, il y a en effet... un chômeur. Un chômeur sur mille personnes citées par le journal, alors qu'il y avait, au moment de l'étude, 40 chômeurs pour 1000 Français $^{16}$. La pauvreté, ou tout au moins une certaine détresse, puisqu'il n'est pas question ici de RMI ou de minimum vieillesse par exemple, est donc quarante fois moins visible dans le journal que dans la vie. Aux difficultés, que l'on veut sans doute croire passagères bien qu'elles conduisent à l'exclusion sociale de certains, la presse préfère les manifestations sportives et associatives, qui rassemblent tout le monde.

\section{CONCLUSION : L’ATTENTION DÉTOURNÉE}

Faite pour une bonne part d'informations-services et de compte-rendus factuels, pour une autre part d'articles valorisant des actions bonnes pour le pays ou les "héros d'un jour», pour reprendre l'expression de Gabriel Ringlet, et enfin d'une très petite minorité d'enquêtes, de portraits ou de dossiers fouillés, la presse locale ne parait être capable de saisir la vie locale que comme une suite ininterrompue de micro-événements sans contexte. La relation que chacun entretient avec ses territoires géographiques ou symboliques, qui est le résultat d'un processus toujours recommencé et jamais d'un déterminisme figé, ne peut donc que lui échapper.

Dans les domaines de la culture et du social, cette incapacité de la presse locale à sortir de l'«événement» est criante. Un spectacle est en général annoncé, et parfois un compte-rendu de la représentation est publié. Mais le journal local ne rend jamais compte du processus de création, même pour les artistes locaux qu'il serait pourtant facile de suivre régulièrement. Pour ce qui est du social, on rend compte des grèves, des conflits sociaux, des licenciements collectifs. Mais le travail lui-même, la vie quotidienne dans les entreprises ou les administrations, n'est jamais traité, ce qui rend les conflits beaucoup plus difficiles à comprendre et renforce l'impression de brutalité qu'ils donnent

15. LE BOHEC, 1998, p. 196.

16. Chiffre trouvé dans un des journaux de notre corpus, Le Télégramme du 25 janvier 2005. 
quand ils surgissent dans les pages du journal. Le social - comme la culture et ses intermittents - se présente ainsi comme un volcan sans sismographe ni vulcanologue. Quand il y a une éruption, tout le monde est surpris. Mais ce n'est pas parce que l'éruption était imprévisible. C'est parce qu'on regardait ailleurs.

Ces deux exemples illustrent ce que Maurice Mouillaud et Jean-François Tétu ont nommé «le standard du fait». Pour eux, il faut revenir sur l'opposition supposée fondamentale entre, d'un côté, le réel (les faits, matériels et chronologiques), et, de l'autre, l'information (construite, mise en forme et diffusée). $\mathrm{Au}$ contraire, il y aurait une «idéologie spontanée des professionnels » qui construit un certain modèle de l'événement - seuls les événements conformes à ce modèle pouvant ensuite devenir des informations. «Les événements médiatiques s'emboîtent dans des formes qui sont déjà des constructions de l'espace et du temps», affirme Maurice Mouillaud. En somme, «tout se passe comme si le journal était écrit sur deux registres : une surface donnée à lire - et un stock de paradigmes tenus en réserve à la manière d'une archive. ${ }^{17}{ }^{17}$ 'est ce stock de paradigmes qui définit le standard du fait. Il inclut notamment la vision institutionnelle et temporellement morcelée de la société que l'on retrouve dans la presse régionale. Ce qu'il faut comprendre ici, c'est que la façon dont un journaliste décrit un fait n'est pas seulement déterminée par ce fait lui-même. De manière plus profonde, les représentations inconscientes que les journalistes se font de ce qu'est un événement et l'endroit où ils se placent dans le champ social pour l'observer ont un rôle aussi grand dans la façon qu'ils vont avoir de le décrire. En un certain sens, il ne peut se passer que ce qui est attendu. Ce qu'on attend de la vie locale, ce que l'on peut accepter d'elle, ce sont des fêtes, des réunions, des spectacles, de temps à autre un procès. Mais pas des processus, lents et sans cesse remis en question, de construction des identités, de relation au territoire ou de développement des individus et des groupes sociaux.

L'hypothèse d'un décalage entre le monde que la presse locale donne à lire et celui que vivent ses lecteurs est donc largement confirmée. L'essoufflement des ventes de la presse régionale pourrait y trouver une de ses explications. Ce décalage paraît en effet assez récent: il date d'une trentaine d'années au plus, c'est-à-dire du moment où la presse régionale connaît ses premières difficultés et essuie les premières critiques. Alors qu'auparavant, presse et société ont toujours évolué en parallèle et en 
interaction - ce qui avait d'ailleurs largement profité au développement économique de la presse régionale.

Plus en détail, cette analyse montre que, malgré son apparente diversité, l'information locale est très hiérarchisée, souvent neutre mais volontiers complaisante, presque jamais critique. Comme bloquée dans le modèle consensuel qui avait fait son succès il y a un siècle, elle répond très bien aux quatre propriétés de l'information locale définies par Jean-François Tétu. L'information locale est rassurante : elle englobe l'individu dans un groupe et parle majoritairement de la fête, mais pas du conflit. Elle est démonstrative : elle révèle et grossit les structures d'un quartier ou d'un village, mais dissimule le travail en profondeur. Elle est banale, c'est-à-dire que, ne retenant « de toute chose que son succès, elle rend totalement banal l'événement extraordinaire ${ }^{18}{ }^{18}$. Elle est enfin promotionnelle : elle se situe dans l'institution permanente de micro-structures et de notabilités locales. La structure de l'information locale apparaît ainsi comme orientée par une vision du monde qui privilégie la stabilité et la tranquillité sociales. Or le monde est complexe, fait d'interrelations qui opèrent en permanence et sur la longue durée. Tout comme l'espace public et le territoire sont marqués par une dimension dynamique essentielle, la façon dont chacun construit son opinion ou son rapport au territoire est le résultat d'un processus en constante recomposition. Mais face à cela, la presse locale ne retient qu'une information superficielle faite d'une juxtaposition de micro-événements sans contexte, sans cause ni conséquence. En somme, elle détourne le regard et l'attention de la profondeur et de la diversité de la société pour l'attirer sur une construction simplifiée à l'extrême des rapports sociaux. Le principal mécanisme à l'œuvre dans la presse régionale nous semble donc être celui de faire diversion.

18. TÉTU, 1995, p. 293. 
ALBERT P. (s.d. a), "Presse », Encyclopadia Universalis, édition électronique V. 10 .

ALBERT P. (s.d. b), " Presse - Naissance et développement de la presse écrite », Encyclopaedia Universalis, Edition électronique vol. 10.

CHARON J.-M. (2003), Les Médias en France, Paris, La Découverte.

DDM - Direction du développement des médias (2007), Tableaux statistiques de la presse édition 2007, Paris, La Documentation française.

JEANNENEY J.-N. (1998), Une histoire des médias, Paris, Seuil.

LE BOHEC J. (1998), «La question du "rôle démocratique" de la presse locale en France », Hermès, n 26-27, Paris, CNRS Editions, p. 185-198.

MARTIN M. (2002), La Presse régionale, Paris, Fayard.

MOUILlAUD M. et TÉTU J.-F. (1989), Le Journal quotidien, Lyon, Presses universitaires de Lyon.

OJD (2006a), $16^{e}$ Observatoire de la presse, Paris, http://www.ojd. com/dyn/_files / downloads/observatoire_16_gp.pdf

OJD (2006b), Book Presse payante grand public 2006, Paris, http://www.ojd .com / dyn/_files/teledocs/124-Book_Presse_Payante_GP_2006.pdf

PAILliART I. (1994), Les Territoires de la communication, Grenoble, Presses universitaires de Grenoble.

RINGLET G. (1981), Le Mythe au milieu du village, comprendre et analyser la presse locale, Bruxelles, Vie ouvrière.

RUELLAN D. (2001), "Guides de villes et sites municipaux », DAMIAN B., RINGOOT R., THIERRY D., RUELLAN D. (dir.), information.loc@l, Le paysage médiatique régional à l'ère électronique, Paris, L'Harmattan, p. 203-218.

TÉTU J.-F. (1995), «L'espace public local et ses médiations », Hermès, n 17-18, Paris, CNRS Editions, p. 287-298. 